\title{
Assistência obstétrica e complicações graves da gestação na América Latina e Caribe: análise das informações obtidas a partir de inquéritos demográficos de saúde
}

\author{
João Paulo Souza, ${ }^{1}$ Mary Angela Parpinelli, ${ }^{1}$ Eliana Amaral ${ }^{1}$ \\ e Jose Guilherme Cecatti ${ }^{1}$
}

Como citar Souza JP, Parpinelli MA, Amaral E, Cecatti JG. Assistência obstétrica e complicações graves da gestação na América Latina e Caribe: análise das informações obtidas a partir de inquéritos demográficos de saúde. Rev Panam Salud Publica. 2007;21(6):396-401.

RESUMO Objetivos. Compilar, consolidar e analisar as informações obtidas por inquéritos do projeto MEASURE DHS acerca de assistência obstétrica e complicações da gestação na América Latina e Caribe.

Métodos. O presente estudo exploratório incluiu sete inquéritos demográficos realizados na década de 1990 (Bolívia, Brasil, Colômbia, Guatemala, Nicarágua, Peru e República Dominicana). Além do levantamento das características das entrevistadas e da assistência obstétrica recebida, foi estimada a ocorrência de complicações (trabalho de parto prolongado e complicações hemorrágicas, hipertensivas e infecciosas).

Resultados. A mediana do número de visitas de pré-natal oscilou entre 4,7 (Bolívia) e 6,6 (República Dominicana). Na Bolívia, Peru e Guatemala foram observadas altas taxas $(>40 \%)$ de assistência ao parto por parteiras tradicionais, parentes e outras pessoas sem treinamento formal. República Dominicana e Brasil apresentaram as maiores taxas de parto em estabelecimento de saúde (>90\%). Na Guatemala, Peru e Bolivia, mais de $45 \%$ dos partos foram domiciliares. A maior taxa de cesárea foi registrada no Brasil (36,4\%); as menores taxas foram registradas no Peru e Guatemala $(<12 \%)$. A taxa de complicações da gestação referidas pelas mulheres foi de 16,7\% no Brasil, 17,9\% na Guatemala, 42,1\% na Colômbia, 42,5\% na Nicarágua, 43,0\% na República Dominicana, 51,7\% na Bolívia e 51,8\% no Peru.

Conclusão. A ocorrência relatada de complicações graves da gestação nos inquéritos avaliados está muito acima da taxa de 15\% citada na literatura, podendo ter sido superestimada. A validação prévia dos questionários utilizados para coleta de dados nesse tipo de estudo é extremamente importante para gerar dados mais adequados.

Palavras-chave Morbidade, complicações na gravidez, inquéritos de morbidade, América Latina, região do Caribe.

1 Universidade Estadual de Campinas, Faculdade de Ciências Médicas, Departamento de Tocoginecologia. Enviar correspondência a João Paulo Souza no seguinte endereço: Rua Alexander Fleming 101, CEP 13083-970, Campinas, SP, Brasil. Fone: +55-193521.9482; e-mail: souzajpd@unicamp.br
No ano 2000, os estados-membros das Nações Unidas assinaram a Declaração do Milênio, que apresenta, como uma de suas metas, a redução em três quartos da razão de morte materna até
2015. A razão de morte materna, um dos principais indicadores da saúde da mulher, representa o número de mulheres que morrem em decorrência de complicações relacionadas à ges- 
tação por 100000 nascidos vivos. $\mathrm{Na}$ América Latina e Caribe, estima-se que ocorram cerca de 22000 mortes maternas por ano, e a razão de morte materna é estimada em 190 mortes por 100000 nascidos vivos (1). Para o cumprimento das metas da Declaração do Milênio, foi proposta uma ampla gama de ações, atualmente em diferentes graus de implementação (2). Monitorar o impacto produzido por essas ações é um desafio em si, pois a obtenção de estimativas confiáveis de mortalidade materna é uma tarefa complexa e custosa $(3,4)$.

Nesse contexto, um grande esforço tem sido realizado para desenvolver e validar outros indicadores da qualidade da saúde materna, como os indicadores de processo e os indicadores indiretos da mortalidade (5). Entre essas abordagens, destaca-se o estudo da morbidade materna grave-por exemplo, os casos de eclâmpsia e hemorragia maciça, entre outros. Essas complicações agudas e graves relacionadas à gestação são mais freqüentes do que o óbito materno, compartilhando muito de seus determinantes. Mais além, as sobreviventes dessas condições (maternal near miss) podem informar diretamente sobre as dificuldades e os obstáculos que tiveram de superar para receber seu tratamento (6-8).

Por outro lado, ainda não foi desenvolvido um sistema de vigilância integrado, com base hospitalar e ampla cobertura geográfica, que monitore a ocorrência de morbidade materna grave. Mesmo nos países com elevadas taxas de institucionalização da atenção ao nascimento, não existe esse tipo de sistema. Nos locais onde a cobertura de serviços é baixa ou variável, e onde a integração de informações hospitalares é ainda incipiente, a realização de inquéritos populacionais pode ser a única maneira de se obter informações populacionais sobre a prevalência de complicações e morbidade materna grave (9).

Nos últimos 15 anos, uma série de estudos demográficos de saúde que abordam (entre outros aspectos) as complicações da gestação e do parto vêm sendo realizados na América Latina e Caribe (10). O programa MEASURE (Monitoring and Evaluation to Assess and
Use Results, ou seja, monitoramento e avaliação para estimar e usar resultados), da Agência Norte-Americana para Desenvolvimento Internacional (USAID), reúne e disponibiliza esses dados para utilização científica. São inquéritos domiciliares com amostragem geralmente entre 5000 e 30000 domicílios, o que confere a eles representatividade nacional. Normalmente, são realizados a cada 5 anos nos países em desenvolvimento. Dessa forma, o presente estudo foi desenvolvido com o objetivo de compilar, consolidar e analisar as informações obtidas por esses inquéritos acerca de assistência obstétrica e complicações da gestação na América Latina e Caribe, de forma a contribuir para a reflexão sobre mortalidade materna nessa região.

\section{MATERIAIS E MÉTODOS}

O presente estudo exploratório consiste na análise secundária de inquéritos demográficos realizados na América Latina e Caribe na segunda metade da década de 1990.

Para fins de seleção, o conjunto de bancos de dados do projeto MEASURE DHS (10) foi consultado na busca de inquéritos demográficos de saúde realizados no continente americano com informações sobre complicações da gestação e parto. Os inquéritos demográficos que não abordavam temas relacionados com a ocorrência de complicações da gestação não foram incluídos nesta análise. Cada um dos 33 inquéritos inicialmente selecionados foi checado para a presença dos dados de interesse de forma manual e eletrônica.

As complicações estudadas nos inquéritos avaliados foram: trabalho de parto prolongado, complicações hemorrágicas, complicações hipertensivas e complicações infecciosas. Essas são as complicações normalmente avaliadas nos inquéritos demográficos, por serem as principais causas de morte materna nos países em desenvolvimento (9). Os dados sobre complicações foram obtidos diretamente das mulheres entrevistadas através da aplicação de um questionário estrutu- rado e referem-se a gestações ocorridas nos 5 anos que precederam a realização do inquérito.

Foi acessado o inquérito mais recente realizado em cada país, obtendose os dados referentes às características das mulheres entrevistadas, da assistência obstétrica e das complicações referidas. A realização de metanálise ou outros procedimentos estatísticos foi considerada inapropriada devido à heterogeneidade entre os estudos e ao fato de que os estudos primários trazem dados oriundos de contextos específicos, com bases populacionais diferentes e estratégia variável de validação dos questionários utilizados. A consulta aos bancos de dados e o manejo das informações obtidas foram realizados através da ferramenta DHS STATcompiler e de planilhas geradas pelo software Excel.

A presente avaliação, por se tratar de análise secundária de banco de dados de mulheres não identificadas, segue todos os princípios de ética contidos na Declaração de Helsinque, preservando a confidencialidade das fontes de informação.

\section{RESULTADOS}

Foram examinados 33 inquéritos demográficos de saúde realizados pelo projeto MEASURE DHS na América Latina e Caribe em busca de informações referentes à ocorrência de complicações na gravidez. Desse total, oito estudos apresentavam informações sobre complicações da gestação e parto (11-18). Na Guatemala, no período analisado, foram realizados dois inquéritos, tendo sido incluído na presente análise apenas o mais recente. Dessa forma, foram analisados sete inquéritos, realizados nos seguintes países: Bolívia, Brasil, Colômbia, Guatemala, Nicarágua, Peru e República Dominicana.

A tabela 1 apresenta as características gerais dos estudos demográficos de saúde incluídos, enquanto que a tabela 2 apresenta as características das mulheres entrevistadas. Observase uma alta taxa de resposta das mulheres, de maneira geral acima de $90 \%$, 
TABELA 1. Características dos inquéritos demográficos de saúde por país, América Latina e Caribe, década de 1990

\begin{tabular}{|c|c|c|c|c|c|c|c|}
\hline Características (n) & $\begin{array}{c}\text { Bolívia, } \\
1998\end{array}$ & $\begin{array}{c}\text { Brasil, } \\
1996\end{array}$ & $\begin{array}{c}\text { Colômbia, } \\
1995\end{array}$ & $\begin{array}{c}\text { República } \\
\text { Dominicana, } \\
1996\end{array}$ & $\begin{array}{c}\text { Guatemala, } \\
1998 / 1999\end{array}$ & $\begin{array}{l}\text { Nicarágua, } \\
1997 / 1998\end{array}$ & $\begin{array}{l}\text { Peru, } \\
1996\end{array}$ \\
\hline Domicílios encontrados & 12281 & 14252 & 11297 & 9026 & 5972 & 11726 & 28805 \\
\hline Mulheres elegíveis & 11831 & 14579 & 12086 & 9034 & 6756 & 14807 & 31241 \\
\hline Mulheres entrevistadas & 11187 & 12612 & 11140 & 8422 & 6021 & 13634 & 28951 \\
\hline Taxa de resposta (\%) & 94,6 & 86,5 & 92,2 & 93,2 & 89,1 & 92,1 & 92,7 \\
\hline
\end{tabular}

Fonte: MEASURE DHS (10).

TABELA 2. Características das mulheres entrevistadas por paísa inquéritos demográficos de saúde na América Latina e Caribe, década de 1990

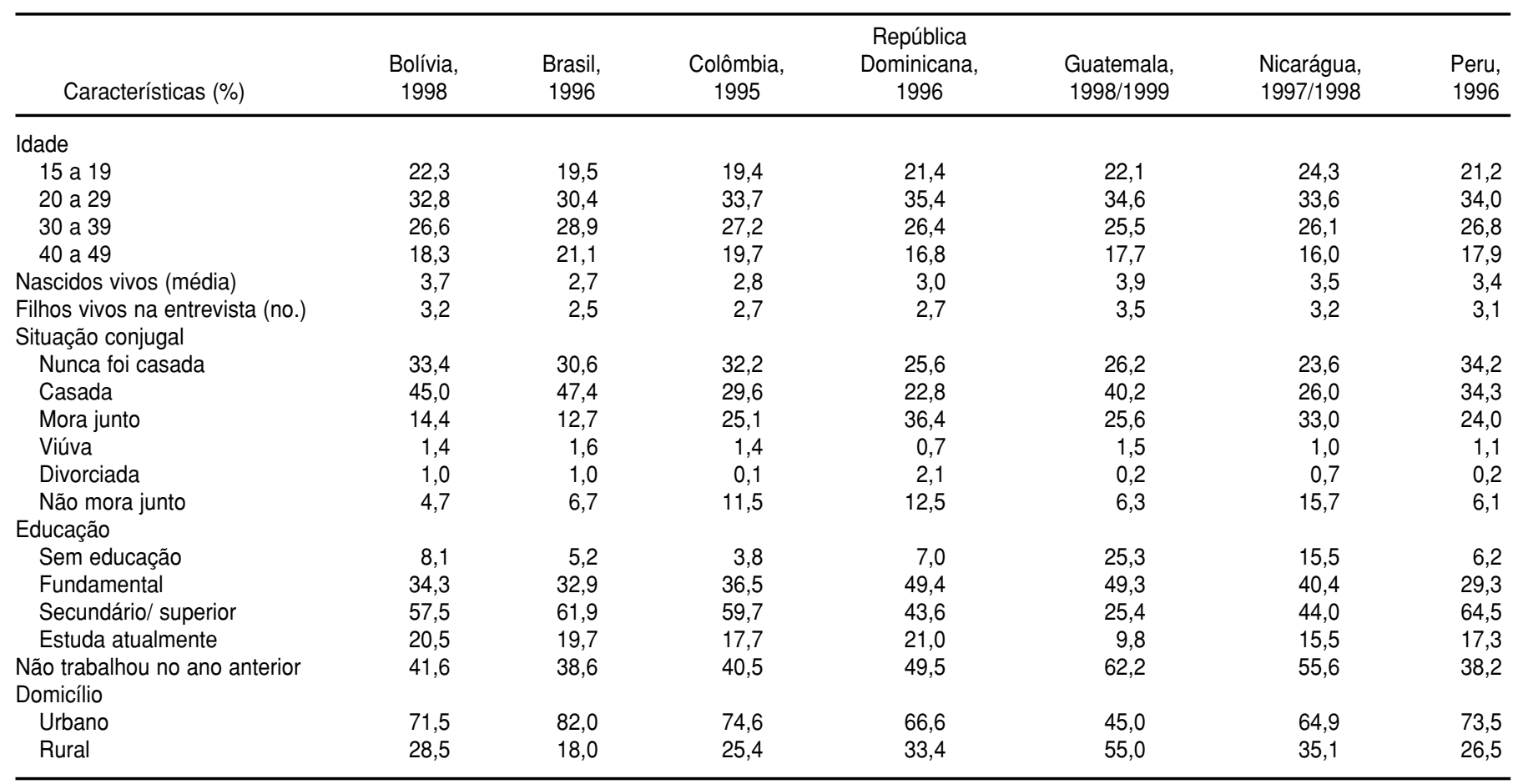

Fonte: MEASURE DHS (10).

a Bolivia: $n=11$ 187; Brasil: $n=12$ 612; Colômbia: $n=11$ 140; Guatemala: $n=6$ 021; Nicarágua: $n=13$ 634; Peru: $n=28$ 951; República Dominicana: $n=8422$.

exceto no estudo realizado no Brasil $(86,5 \%)$ e na Guatemala $(89,1 \%)$. A média de nascidos vivos foi superior a 3,0 por mulher, exceto no caso do Brasil e Colômbia, onde essa média foi menor (2,7 e 2,8, respectivamente).

As características da assistência obstétrica nos 5 anos que precederam os estudos são descritas na tabela 3 . A mediana do número de visitas de pré-natal oscilou entre 4,7 e 6,6, sendo menor na Bolívia e maior na República Dominicana e no Brasil. A mediana do número de meses de gestação no momento da primeira visita de pré-natal ficou ao redor de 3,0. A assistência pré-natal esteve a cargo principalmente de profissionais médicos na Bolívia, Brasil e Colômbia, enquanto que na República Dominicana e Nicarágua esse papel esteve preponderantemente a cargo de outros profissionais de saúde. Excetuando-se a Guatemala, o papel desempenhado pelas parteiras foi pouco expressivo.
Uma parcela importante de mulheres não recebeu atenção pré-natal, principalmente na Bolívia e Peru (>30\%). A menor taxa de ausência de assistência pré-natal foi registrada na República Dominicana (1,5\%). Na Bolívia, Guatemala e Peru foram observadas altas taxas-41,7, 57,3 e $42,4 \%$, respectivamente-de assistência ao parto por pessoas sem treinamento formal (parteiras tradicionais, parentes e outros). Na República Domini- 
TABELA 3. Características da assistência obstétrica nas gestações que resultaram em nascidos vivos ${ }^{a}$ nos 5 anos que precederam 0 inquérito, América Latina e Caribe, década de 1990

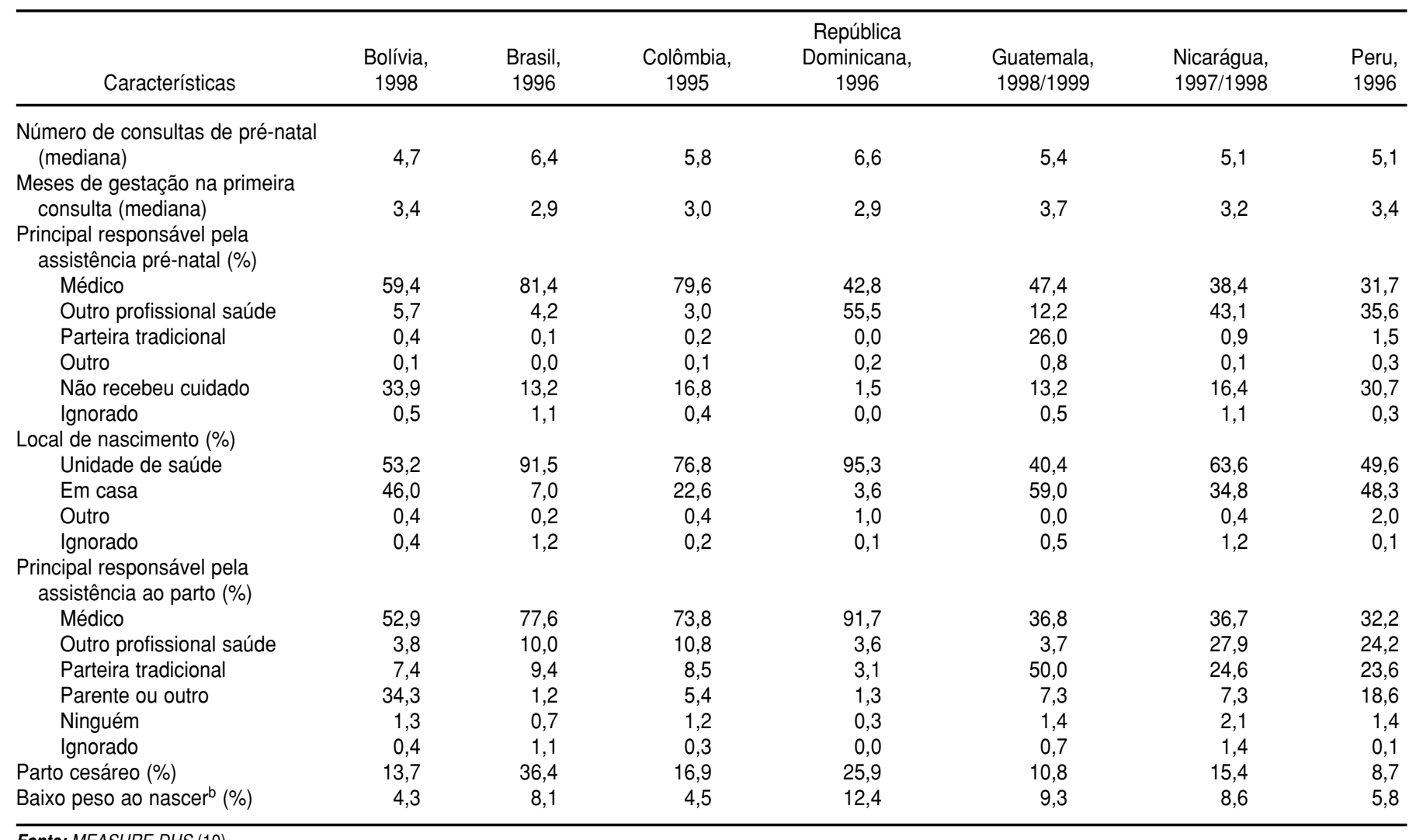

Fonte: MEASURE DHS (10).

${ }^{a}$ Nascidos vivos nos últimos 5 anos: Bolívia $=6$ 893; Brasil $=4$ 782; Colômbia $=5$ 050; Guatemala $=4$ 545; Nicarágua = $7992 ;$ Peru $=15$ 639; República Dominicana $=4$ 379.

b Referido pela mulher; peso ao nascer < $2500 \mathrm{~g}$.

cana, Brasil e Colômbia, foram observadas altas taxas de assistência ao parto prestada por médicos. República Dominicana e Brasil apresentaram as maiores taxas de parto em estabelecimentos de saúde, enquanto que uma elevada porcentagem de partos domiciliares foi observada na Guatemala, Peru e Bolívia. A maior taxa de parto cesáreo foi observada no Brasil (36,4\%), enquanto que as menores taxas foram observadas na Guatemala e Peru (10,8 e 8,7\%). Bolívia e Colômbia apresentaram as menores taxas de baixo peso ao nascer (4,3 e 4,5\%), enquanto que a República Dominicana apresentou a maior taxa $(12,4 \%)$.

Brasil e Guatemala apresentaram as menores taxas de complicações referidas pelas mulheres, respectivamente 16,7 e $17,9 \%$. Por outro lado, Bolívia e Peru apresentaram as taxas mais elevadas, respectivamente 51,7 e 51,8\%.
Para os outros países, as taxas de complicações referidas pelas mulheres foram as seguintes: Colômbia, 42,1\%; Nicarágua, 42,5\%; e República Dominicana, $43,0 \%$.

A tabela 4 apresenta a distribuição das complicações citadas pelas mulheres para os partos ocorridos até 5 anos antes do inquérito. A porcentagem de trabalho de parto prolongado foi muito elevada na Bolívia $(41,0 \%)$ e no Peru $(34,6 \%)$. À exceção de Brasil e Guatemala, foram observadas taxas elevadas de sangramento excessivo nos demais países. A porcentagem de convulsões referidas foi relativamente alta, principalmente nos estudos realizados na Bolívia $(8,7 \%)$ e Peru (7,4\%). De modo geral, dentre os países avaliados, Brasil e Guatemala apresentaram os valores globalmente mais baixos para todas as complicações referidas pelas mulheres entrevistadas.

\section{DISCUSSÃO}

A análise dos dados de sete inquéritos demográficos de saúde realizados na América Latina e Caribe revelou uma taxa elevada de morbidade materna referida que, na maioria dos países estudados, foi superior a 40\%. Em contraposição a esse achado, acreditase que em torno de $15 \%$ das gestantes possam desenvolver complicações obstétricas de alguma gravidade, necessitando atendimento hospitalar (3, 5). Dentre os inquéritos analisados, apenas dois relataram uma taxa próxima ao esperado (Brasil 16,7\% e Guatemala 17,9\%). As porcentagens demasiadamente elevadas de complicações referidas pelas mulheres nos demais estudos demográficos realizados na América Latina e Caribe sugerem que pode ter havido uma supervalorização de problemas menores. Embora os in- 
TABELA 4. Porcentagem de complicações em gestações que resultaram em nascidos vivos ${ }^{\mathrm{a}}$ nos 5 anos que precederam 0 inquérito, América Latina e Caribe, década de 1990

\begin{tabular}{|c|c|c|c|c|c|c|c|}
\hline Complicações $^{b}$ & $\begin{array}{c}\text { Bolívia, } \\
1998\end{array}$ & $\begin{array}{c}\text { Brasil, } \\
1996\end{array}$ & $\begin{array}{c}\text { Colômbia, } \\
1995\end{array}$ & $\begin{array}{c}\text { República } \\
\text { Dominicana, } \\
1996\end{array}$ & $\begin{array}{l}\text { Guatemala, } \\
1998 / 1999\end{array}$ & $\begin{array}{l}\text { Nicarágua, } \\
1997 / 1998\end{array}$ & $\begin{array}{l}\text { Peru, } \\
1996\end{array}$ \\
\hline Sangramento excessivo & 22,3 & 5,4 & 25,7 & 25,1 & 7,4 & 28,3 & 34,6 \\
\hline Infecção vaginal & 15,3 & 3,7 & 4,9 & 6,1 & 3,9 & 7,7 & 12,2 \\
\hline Convulsões & 8,7 & 2,7 & 2,1 & 5,2 & 1,7 & 3,3 & 7,4 \\
\hline Nenhuma & 48,3 & 83,3 & 57,9 & 57,0 & 82,1 & 57,5 & 48,2 \\
\hline
\end{tabular}

Fonte: MEASURE DHS (10)

a Nascidos vivos nos últimos 5 anos: Bolívia = 6 893; Brasil = 4 782; Colômbia $=5$ 050; Guatemala = 4 545; Nicarágua = $7992 ;$ Peru = 15 639; República Dominicana = 4 379.

b Algumas mulheres referiram a ocorrência de mais de uma complicação.

quéritos demográficos de saúde sejam desenvolvidos dentro de uma sistemática bem estabelecida (10), a estratégia de validação dos questionários utilizados não é ainda uniforme. Por outro lado, na literatura podem ser identificados estudos de validação de questionários de morbidade materna grave com resultados conflitantes, sendo ainda incerta a evidência sobre quão acurada é a história de gestação e parto contada pelas mulheres $(19,20)$.

Os inquéritos demográficos de saúde têm no viés de recordação uma característica implícita, uma vez que buscam identificar eventos ocorridos num período relativamente longo, em torno de 5 anos. Além desse viés, outros fatores podem influenciar a sua acurácia, como os aspectos sociais e culturais que adicionam complexidade ao processo saúde-doença e sua percepção. No caso de distocias associadas ao trabalho de parto prolongado, limiares dolorosos diferenciados individualizam a experiência temporal, alongando ou diminuindo a sua percepção. Os questionários também transferem, pelo menos em parte, a responsabilidade diagnóstica para o respondente, fato que pode elevar a freqüência de diagnósticos impróprios. No caso das convulsões, desmaios, calafrios e outros tremores podem ser confundidos, o que ajudaria a explicar uma porcentagem de convulsões referidas tão alta quanto $8,7 \%$ (11). De maneira análoga, os sinais e sintomas compatíveis com infecções do trato reprodutivo (febre, dor e corrimento fétido, entre outros) e mesmo de hemorragia (como sangramento abundante, fraqueza, desmaios) podem ser confundidos com outros quadros que ocorrem comumente no puerpério.

Em demografia, a teoria da transição demográfica descreve a redução das taxas de natalidade e de mortalidade como parte do desenvolvimento socioeconômico de um país (21). Os achados do presente estudo ilustram o intrincado relacionamento existente entre as condições sociodemográficas e as condições para assistência à mulher durante a gestação e o parto. Assim, foi possível o reconhecimento de dois padrões sociodemográficos distintos e opostos de populações femininas: o primeiro é caracterizado por uma transição demográfica mais adiantada, com mulheres de maior idade, menor fertilidade, maior educação, maior atividade econômica e maior urbanização. $\mathrm{O}$ outro padrão caracteriza-se pelo inverso: maior fertilidade, menor educação, menor atividade econômica e menor urbanização. Quando as condições de assistência obstétrica são analisadas sob a perspectiva sociodemográfica, observa-se que, nos países onde a transição demográfica é mais evidente (por exemplo, Brasil), a assistência obstétrica caracteriza-se por ser institucionalizada, com atenção pré-natal de início mais precoce e assistência pré-natal e ao parto essencialmente providas por profissionais de saúde.

Por outro lado, nos países em que os sinais de transição demográfica são menos evidentes (por exemplo, Guate- mala), a assistência obstétrica é menos profissionalizada e menos institucionalizada. Há ainda expressiva porcentagem de mulheres que recebe assistência pré-natal e ao parto de parteiras tradicionais ou outras pessoas não qualificadas, além de um início mais tardio do pré-natal. Esses achados, que sugerem o compartilhamento de determinantes entre a transição demográfica e a assistência obstétrica mais qualificada, reforçam a necessidade de ações que ultrapassam em muito o campo estrito da saúde para a efetiva melhora das condições de saúde materna.

Concluindo, observamos padrões de assistência obstétrica que refletem o grau de desenvolvimento socioeconômico e de transição demográfica. Ademais, os achados do presente estudo sugerem que os inquéritos demográficos de saúde avaliados podem estar superestimando a ocorrência de complicações da gestação, e é possível que tais inquéritos não representem a real ocorrência de graves complicações maternas associadas à gestação e ao parto. Entre outros, existe uma subrepresentação das complicações ocorridas mais precocemente na gestação e daquelas relacionadas a abortamento e que resultaram em óbito fetal. Para um aproveitamento mais adequado das informações colhidas, sugere-se que os questionários de morbidade materna usados nos próximos inquéritos demográficos sejam previamente validados e, se necessário, que sejam desenvolvidos fatores de correção para ajuste das estimativas obtidas. 


\section{REFERÊNCIAS}

1. World Health Organization. Maternal mortality in 2000: estimates developed by WHO, UNICEF, and UNFPA. Genebra: WHO; 2003. Disponível em: http://www.reliefweb.int/ library/documents/2003/who-saf-22oct.pdf. Acessado em junho de 2007.

2. United Nations. United Nations Millennium Declaration. Nova Iorque: UN; 2000. Disponível em: http://www.un.org/millennium/ declaration/ares552e.htm. Acessado em 22 de abril de 2007.

3. World Health Organization, United Nations Children's Fund, United Nations Population Fund. Guidelines for monitoring progress in the reduction of maternal mortality. Nova Iorque: UNICEF; 1997.

4. World Health Organization. Indicators to monitor maternal health goals. Genebra: WHO; 1994.

5. Maine D, Akalin M, Ward V, Kamara A. The design and evaluation of maternal mortality programs. Nova Iorque: Center for Population and Family Health, Columbia School of Public Health; 1997.

6. Pattinson RC, Hall M. Near misses: a useful adjunct to maternal death enquiries. Br Med Bull. 2003;67(1):231-43.

7. Souza JP, Cecatti JG, Parpinelli MA, de Sousa $\mathrm{MH}$, Serruya SJ. Revisão sistemática sobre morbidade materna near miss. Cad Saude Publica. 2006;22(2):255-64.

8. Weeks A, Lavender T, Nazziwa E, Mirembe F. Personal accounts of 'near-miss' maternal mortalities in Kampala, Uganda. BJOG. 2005; 112(9):1302-7.

9. United Nations Population Fund. Maternal mortality update 2002 - a focus on emer- gency obstetric care. Nova Iorque: UNFPA; 2003.

10. Macro International. MEASURE DHS (Demographic and Health Surveys) project. ORC Macro: Measure DHS, 2006. Disponível em: http://www.measuredhs.com. Acessado em 24 de novembro de 2006.

11. Bolivia. Encuesta nacional de demografía y salud 1998. ORC Macro: Measure DHS STATcompiler, 2007. Disponível em: http:// www.measuredhs.com/pubs/pub_details. cfm?ID=141\&srchTp=type. Acessado em 24 de junho de 2007.

12. Brasil. Pesquisa nacional sobre demografia e Saúde 1996. ORC Macro: Measure DHS STATcompiler, 2007. Disponível em: http:// www.measuredhs.com/pubs/pub_details. cfm?ID=119\&ctry_id=49\&SrchTp=available. Acessado em 24 de junho de 2007.

13. Colombia. Encuesta nacional de demografía y salud 1995. ORC Macro: Measure DHS STATcompiler, 2007. Disponível em: http:// www.measuredhs.com/pubs/pub_details. cfm?ID=106\&srchTp=type. Acessado em 24 de junho de 2007.

14. República Dominicana. Encuesta demográfica y de salud 1996. ORC Macro: Measure DHS STATcompiler, 2007. Disponível em: http://www.measuredhs.com/pubs/pub details.cfm?ID=124\&srchTp=type. Acessado em 24 de junho de 2007.

15. Guatemala. Encuesta nacional de salud materno infantil 1998/99. ORC Macro: Measure DHS STATcompiler, 2007. Disponível em: http://www.measuredhs.com/pubs/pub details.cfm?ID=275\&srchTp=type. Acessado em 24 de junho de 2007.
16. Nicarágua. Encuesta nicaragüense de demografía y salud 1997/98. ORC Macro: Measure DHS STATcompiler, 2007. Disponível em: http://www.measuredhs.com/pubs/pub_ details.cfm?ID=64\&srchTp=type. Acessado em 24 de junho de 2007.

17. Peru. Encuesta demográfica y de salud familiar 1996. ORC Macro: Measure DHS STATcompiler, 2007. Disponível em: http:/ /www. measuredhs.com/pubs/pub_details.cfm? $\mathrm{ID}=129 \&$ srchTp=type. Acessado em 24 de junho de 2007.

18. Guatemala. Encuesta nacional de salud materno infantil 1995. ORC Macro: Measure DHS STATcompiler, 2007. Disponível em: http://www.measuredhs.com/pubs/pub_ details.cfm?ID=112\&srchTp=ctry\&ctry_id= $15 \&$ flag=pub. Acessado em 24 de junho de 2007.

19. Stewart MK, Stanton CK, Festin M, Jacobson $\mathrm{N}$. Issues in measuring maternal morbidity: lessons from the Philippines Safe Motherhood Survey Project. Stud Fam Plann. 1996; 27(1):29-35.

20. Ronsmans C, Achadi E, Cohen S, Zazri A. Women's recall of obstetric complications in south Kalimantan, Indonesia. Stud Fam Plann. 1997;28(3):203-14.

21. Anonymous. Demographic transition in the Americas. Epidemiol Bull. 1994;15(1):9-12.

Manuscrito recebido em 4 de dezembro de 2006. Aceito em versão revisada em 16 de maio de 2007.
ABSTRACT

Obstetric care and severe pregnancy complications in Latin America and the Caribbean: an analysis of information from demographic health surveys
Objective. To compile, consolidate, and analyze information obtained in surveys conducted by the MEASURE DHS [Demographic and Health Surveys] program, concerning obstetric care and pregnancy complications for women in Latin America and the Caribbean, in the five years before the survey.

Methods. This exploratory study utilized data from demographic surveys carried out in the 1990s in seven countries of Latin America: Bolivia, Brazil, Colombia, the Dominican Republic, Guatemala, Nicaragua, and Peru. The study describes the characteristics of the women who were interviewed and of the obstetric care that they received in the five years before the respective survey, and it also estimates the occurrence of prolonged labor and of hemorrhagic, hypertensive, and infectious complications in those five years. Results. The median number of prenatal consultations ranged from 4.7 in Bolivia to 6.6 in the Dominican Republic. More than $40 \%$ of deliveries in Guatemala, Peru, and Bolivia were attended by traditional midwives, relatives, or other persons without formal training. The highest rates of deliveries performed in health care facilities $(>90 \%)$ were in the Dominican Republic and Brazil. In Guatemala, Peru, and Bolivia more than $45 \%$ of deliveries were at home. The highest rate of cesarean delivery was in Brazil (36.4\%), and the lowest rates $(<12 \%)$ were in Peru and Guatemala. The rate of pregnancy complications reported by the women surveyed was $16.7 \%$ in Brazil, $17.9 \%$ in Guatemala, $42.1 \%$ in Colombia, $42.5 \%$ in Nicaragua, $43.0 \%$ in the Dominican Republic, $51.7 \%$ in Bolivia, and $51.8 \%$ in Peru. Conclusion. The reported occurrence of severe pregnancy complications in the surveys we examined was well above the $15 \%$ rate reported in other scientific literature, suggesting that these complications may have been overestimated in the MEASURE DHS surveys. Prior validation of the questionnaires used for data collection is extremely important in the generation of high-quality data. 